\title{
De la Historia de los Alimentos a la Historia de la Nutrición: Reflexiones sobre América Latina en la Primera Mitad del Siglo XX
}

\author{
Joel Vargas Domínguez \\ UNIVERSIDAD NACIONAL AUTÓNOMA DE MÉXICO - CEIICH
}

\section{RESUMEN}

El presente artículo tiene por dos objetivos principales. El primero de ellos es mostrar dónde se insertan los estudios sobre la nutrición en el campo más amplio de los estudios sobre alimentación, así como vincular historiografías sobre alimentos con la historia de la disciplina nutricional. El segundo objetivo es ofrecer una visión panorámica sobre los estudios de la nutrición en algunos países latinoamericanos en la primera mitad del siglo XX. En este panorama, enfatizo algunos procesos históricos paralelos en estos países. Lo anterior me sirve para proponer algunas líneas de investigación para la historia de la nutrición en América Latina en perspectiva comparada.

Palabras clave: historia de la nutrición, nutrición social, eugenesia, historia comparada, América Latina

\begin{abstract}
This paper has two main objectives. The first one is to situate the most recent nutrition studies in the broader field of the food studies movement. By doing this, I try to examine the common threads in diverse historiographies related to food, and the common ties with the history of nutrition. The second objective of the article is to offer an overview of the recent historiography of nutrition in Latin America in the first half of the 20th century. In this overview I emphasize some historical processes common to these countries. By doing so, I propose some possible threads to follow in the history of nutrition in Latin America in comparative perspective.
\end{abstract}

Keywords: history of nutrition, social nutrition, eugenics, compared history, Latin America 


\section{INTRODUCCIÓN}

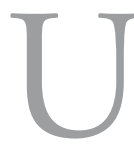

na editorial del Boletín de la Oficina Sanitaria Panamericana (OPS) de 1940 concluía de la siguiente manera: "Con un poco de ciencia, su tantico juicio, y algún discernimiento, todos pueden aprender a comer bien, y hasta a producir, por lo menos en parte lo que comen”. (1940,280) En estas líneas se resumían varios temas y la forma de aproximarse al tema de la alimentación de los médicos miembros de la OPS. Por un lado, resaltaba el papel de la ciencia como necesaria para llevar a cabo una correcta alimentación. Apelaba a una disposición del individuo para tener control de sí mismo en el plano alimentario, a un conocimiento de lo bueno y lo malo en la alimentación, a un juicio moral que le permitía al individuo, corregir sus instintos. Esto asumía que si se dejaba la alimentación en manos de las sensaciones, ésta seria incorrecta. Por otro lado, entendía que en parte el problema de la alimentación era de índole educativa, es decir, el pueblo en sí no era culpable de sus hábitos, sino la falta de educación en el terreno de la nutrición o alimentación racional. Por otro lado, también se recuperaba el tema de la producción de alimentos como parte de la solución.

Estas palabras, publicadas en 1940, nos permiten cuestionarnos, ¿cómo se llegó a entender a la alimentación como un fenómeno científico, moral, y de producción y como un problema internacional? ¿Por qué un organismo como la OPS acometía el trabajo de ofrecer orientaciones para una correcta alimentación? Estas preguntas las podemos responder parcialmente si hacemos un estudio detallado de las políticas internas de la OPS. Sin embargo, también podemos responderlas si entendemos que el estudio de la nutrición era una parte importante de las políticas públicas de salud en los países latinoamericanos. Lo anterior, nos puede llevar a otro cuestionamiento y que es, cuestionar cómo la nutrición se integró a los discursos de alimentación en América Latina.

El presente trabajo es una primera aproximación para tratar de ofrecer respuestas a estos cuestionamientos. Este artículo está dividido en dos grandes secciones. En la primera de ellas, lo que intento hacer es ofrecer una mirada panorámica a los estudios históricos 
sobre alimentación abordados desde una perspectiva transnacional y ubicar a la historia de la nutrición en dichas investigaciones. En la segunda parte, haré un esbozo de algunas tendencias en común que hubo en los proyectos de nutrición latinoamericanos, mismos que me permiten formular una posible respuesta a desde dónde abordar una historia de la nutrición latinoamericana en perspectiva comparada.

Hablar de una visión panorámica implica que ya hay una suficiente cantidad de estudios históricos sobre la alimentación y la nutrición como para enfrentarse a esta tarea. En la primera sección recupero el trabajo de JeffreyM. Pilcher quien es uno de los investigadores sobre la historia de la alimentación en Latino América más reconocidos. En un libro editado por él, The Oxford Handbook of Food History hace una excelente introducción a los estudios sobre alimentación más recientes (Pilcher, 2012). En buena medida, la primera parte de este trabajo se basa en las propuestas temáticas por él elegidas para acercarse al estudio de la alimentación. La segunda sección, recupera trabajos recientes de investigadores en el área de la historia de la nutrición en diferentes países de Latinoamérica para elaborar esbozos del surgimiento de intereses en la nutrición en sus países, para finalmente, en la conclusión, sugerir algunas posibles vías de investigación para abordar la historia de la nutrición en perspectiva comparada desde Latinoamérica.

\section{ACercamientos globales o transnacionales a la historia de}

\section{LA ALIMENTACIÓN}

En un momento en que hay una tendencia para estudiar a los fenómenos sociales desde perspectivas multidisciplinares, el estudio de la alimentación ha cobrado relevancia. Los estudios sobre el tema son objeto de revistas especializadas, compilaciones de libros, seminarios, talleres, conferencias y existe ya un número importante de investigadores que intentan abordar las diferentes aristas de la alimentación, tanto desde una perspectiva histórica como desde una contemporánea. Todos tenemos precedentes en nuestras líneas de investigación y entre ellas, recuperamos frecuentemente 
a Sidney Mintz como uno de los pioneros en hacer una historia sobre alimentación y alimentos tal como las hacemos ahora. Su libro Sweetness and Power (Mintz, 1985), representó uno de los modelos a seguir al analizar el uso de los alimentos como mercancías y relacionar entre otras cosas el auge del imperio británico con el cambio del gusto global hacia lo dulce, con las plantaciones de azúcar en el Caribe y el tráfico de esclavos. El libro orientó los estudios posteriores sobre cómo abordar el nexo entre la producción y el consumo de alimentos, y las transformaciones sociales vinculadas a estos procesos en una escala global, o lo que podríamos llamar imperial.

Por otro lado, la historia social ha abordado el tema de la alimentación, principalmente desde el punto de vista de los grupos que tienen carencias alimenticias, que padecen hambre, y que sirven de marco explicativo de muchas de las luchas sociales que han sucedido a lo largo de la historia. Estos grupos, los trabajadores, campesinos, obreros, mujeres, sindicalistas, y muchos otros más, sirven para quienes hacemos historia de la alimentación como actores indispensables de las historias que contamos. (Aguilar Rodríguez, 2008; Drinot, 2016; Remedi, 2006; Turner, 2014). Muchas de las luchas sociales se han articulado pensando en la carencia de alimentos como detonadores de movimientos sociales, sin cuestionar el por qué existe la ausencia de alimentos. Ante ello, existe lo que se conoce como la paradoja de la abundancia, es decir, desabasto o ausencia de alimentos en países ricos en recursos naturales, como los países latinoamericanos. (Levenstein, 2003). Lo anterior puede ser explicado en parte gracias al trabajo de Amartya Sen, quien explica cómo la falta de alimentos, no es fruto de escasez, sino de una falta de distribución adecuada (Sen, 1981). Lo cual nos lleva a cuestionar de manera más amplia el poder explicativo que atribuimos a los alimentos y su producción y distribución. Al hacer esto, podemos cuestionar narrativas históricas y actuales que justifican la necesidad de una mayor producción para acabar con el problema de la mala alimentación, narrativas que tienen un trasfondo histórico importante y que debe ser considerado. Quizás 
hay que indagar en la historia sobre cómo surgió la idea de la producción como explicación y causa del hambre, no solo dentro de los contextos nacionales sino desde de un acercamiento global.

Por otro lado, en la mayoría de las narrativas sobre alimentación, no se cuestiona el uso de un lenguaje científico para referirse a los alimentos. Además, existen pocos trabajos que aborden el vínculo entre la historia de una disciplina como la nutrición con otras historiografías, como la laboral, la social o la económica. Podemos cuestionar, por ejemplo cómo fue recibido el conocimiento nutricional por diferentes actores sociales, como obreros o campesinos, amas de casa, entre otros. También podemos dilucidar cuáles fueron los intereses de los actores que crearon las instituciones dedicadas al estudio de la alimentación, así como indagar sobre los flujos de dinero que posibilitaron la creación de dichas instituciones. También podemos abordar estos estudios desde una perspectiva de historia de la ciencia o de la medicina, para comprender cómo se entendió la nutrición como parte de las investigaciones necesarias para analizar el cuerpo de los obreros y, siguiendo este hilo, podríamos vincular la necesidad de la investigación sobre alimentación con la construcción de identidades nacionales. El estudio de la alimentación, como se puede observar es muy amplio, y ofrece múltiples vías de investigación, por lo que debemos acotar para que podamos ofrecer perspectivas analíticas que puedan ser de utilidad.

Quizás la dificultad del análisis radica en la naturaleza del objeto de estudio en sí. De acuerdo con Suremain y Katz, desde una perspectiva antropológica, se puede caracterizar a la alimentación "como un conjunto de representaciones, discursos y prácticas en relación con la producción, los intercambios comerciales y no comerciales, la distribución, la preparación y el consumo de productos alimenticios" (Suremain y Katz, 2009) Sin embargo, a la definición anterior le hace falta algo que ha estado vinculado con la alimentación por lo menos en los últimos dos siglos, y que es el estudio de la alimentación desde una perspectiva tecnocientífica, es decir, desde la nutrición. ¿Es importante añadir esta capa de análisis 
a la alimentación? Si, dado que la mayor parte de los análisis de intercambios comerciales, producción de alimentos, consumo y comprensión del fenómeno desde el siglo XIX recupera o se basa en un lenguaje que usa a la ciencia. Pensemos en algún documento relacionado con la alimentación, desde la óptica de cualquier disciplina, que no recupere nociones como requerimientos mínimos, calorías o nutrimentos en algún momento. Inclusive, aún descripciones densas de la práctica antropológica recuperan en algún punto las ideas de "fuente de energía", nociones que al estar asociadas con el conocimiento científico tienden a pasar desapercibidas y ser consideradas "neutras", lo cual, como argumento más adelante, no es cierto. El lenguaje de la nutrición ha permeado quizás más en el lenguaje coloquial que el de otras disciplinas, quizás por los proyectos educativos implementados en la primera mitad del siglo XX, similares a los que apuntaba la editorial de la OPS del inicio del artículo. Es por ello que una descripción adecuada de la alimentación actual debe incluir el estudio científico de la misma.

Ahora bien, en este trabajo me enfocaré en el estudio histórico de la alimentación en Latinoamérica. La mayoría de los estudios sobre alimentación recuperan historias localizadas dentro de los límites que surgen en el siglo XIX, en los Estados nacionales, aunque algunas historias recuperan un punto de vista regional o global. Partiendo de estas dos miradas, unas nacionales y otras que tratan vincular procesos más amplios, en esta primera sección abordaré los estudios de la alimentación desde una perspectiva global o transnacional, dados los intercambios de alimentos y conocimientos sobre la alimentación.

\section{Historias DE ENCUENTROS}

Un buen número de los estudios sobre alimentación, desde esta perspectiva transnacional, se ocupan de los encuentros entre el Viejo y el Nuevo Mundo. A estas narrativas, las llamo historias de encuentros, dada la presencia de dos culturas culinarias distintas que se encuentran. Esta clasificación puede ser ampliada si incorporamos, por ejemplo, estudios de alimentación de cómo ha cambiado la dieta 
de grupos poblacionales ante la incorporación de cambios en las formas de pensar la alimentación. Por ejemplo, podría pensarse en el cambio en el consumo de leche con el auge de este producto como representación de un alimento modelo (Mckee, 1997; Zazueta, 2011).

Uno de los primeros y más importantes trabajos sobre estos encuentros es el ya mencionado de Sidney Mintz del comercio trasatlántico de azúcar y cómo este comercio no puede entenderse sin el cambio en los patrones de consumo. Sin embargo, otro libro abrió la puerta al estudio global de los alimentos, Alfred W. Crosby Jr. (Crosby, 1972) en The Columbian Exchange, exploró el encuentro entre el Viejo y el Nuevo Mundo, aunque enfocado en el intercambio de especies animales y vegetales y su impacto ambiental. Entre este impacto, se encuentra el de los alimentos, y de alguna manera explica cómo cambió el mundo culinario entre colonizados y colonizadores. Partiendo de este encuentro, Rebeca Earle expuso en su libro The Body of the Conquistador cómo la alimentación durante el periodo del encuentro se entendía como un elemento que podía tener un influjo negativo o positivo en los individuos y las poblaciones. Los primeros colonizadores europeos de lo que sería llamado el territorio americano consideraron que el cambio de dieta tenía efectos negativos sobre su salud, y pensaron en la dieta europea como un contrapeso al efecto nocivo del ambiente y los alimentos nativos. En este temprano periodo colonial, se consideró que la comida era, "más que otra cosa, lo que hacía a los cuerpos europeos diferentes de los indígenas”. Desde este periodo, la alimentación se consideró un elemento degenerador o regenerador, que podía hacer a los europeos indígenas o a los indígenas civilizados (Earle, 2012,pos 1,150; Earle, 2010). El efecto de las dietas "exóticas" sobre los cuerpos civilizados de los colonizadores y conquistadores fue una de las preguntas comunes que se hicieron en varios de los emprendimientos colonizadores, como también ha argumentado Steven Shapin, y se reinventaron e incorporaron nuevas explicaciones y soluciones a lo que se consideró desde muy temprano como el "problema" de la alimentación, pero siempre manteniendo la duda sobre el efecto en 
la "constitución" de los cuerpos de una alimentación nativa (Shapin, 2014). Este argumento explica en parte el fuerte impulso a consumir unos alimentos y no a otros, dependiendo de su origen, y cómo el intercambio de alimentos estuvo marcado por ideas asociadas con el degeneracionismo, la idea de que el ambiente podía cambiar la constitución corporal.

Otras historias de encuentros recuperan en parte uno de los aspectos que, parece obvio, pero que había sido pasado por alto en las explicaciones sobre la alimentación del Nuevo Mundo. El aspecto sensorial. El artículo de Marcy Norton de 2006, (Norton, 2006) que retoma el caso del chocolate, es ilustrador en este aspecto. Existe (o existía) la idea de que el consumo de chocolate, tradicional de Mesoamérica, había sido modificado por los europeos para quitarle el sabor "primitivo", y que su consumo se había popularizado por entender al chocolate como un tratamiento terapéutico. Sin embargo, lo que argumenta Norton, es que el chocolate fue adoptado no como resultado de sus usos medicinales, de su medicalización, sino que el argumento de sus propiedades terapéuticas fue creado para justificar el consumo. Retomando las ideas de Pierre Bourdieu, Norton argumenta que el gusto es de naturaleza contextual, es decir, que no existe un elemento innato en nuestra capacidad de distinguir entre lo que nos gusta o no, sino que es un fenómeno social, cultural y dependiente del momento histórico en que se produjo el intercambio. El deseo de azúcar en el Nuevo Mundo, como lo muestra Mintz, no se basa solo en el sabor, sino en una multiplicidad de factores sociales y culturales. Sin embargo, el sabor quedó oculto en las explicaciones del por qué un alimento es preferido sobre otros, aplastado por explicaciones culturales, o económicas. Lo que hace Norton es recuperar el sabor como uno de los motores históricos del intercambio alimenticio y de los cambios sociales. De una manera similar, Jeffrey Pilcher se encuentra elaborando actualmente una historia de la difusión de algunos alimentos, entre ellos la cerveza mexicana, en el mundo globalizado. Como ha mostrado Pilcher en otros trabajos, es hasta que se industrializa la producción de tortillas, que su sabor es apreciado en el mundo (Pilcher, 2012). Lo cual nos 
refleja un fenómeno asociado con la estandarización y creación de redes de distribución de alimentos más complejas gracias a las modernas técnicas de conservación y el transporte más rápido de un sitio a otro (Pilcher, 2006). Lo mismo ha sucedido con la adquisición de sabores en el mundo global, como muestran otros trabajos (Liu, 2015; Montanari, 2015).

Otra de las características de la alimentación es su capacidad de creación de diferentes tipos y niveles de identidades. Al proponer que existen encuentros de culturas culinarias, de cambios sociales en los hábitos de alimentación, asumimos la creación de los "otros", quienes tienen el patrón culinario distinto. Esta diferenciación, culinaria, social, y que se argumentó inclusive en términos raciales, otorgan a la alimentación el poder de crear identidades, identidades que viajan y se transforman conforme se desplazan los comensales, es decir, estas identidades culinarias pueden mantenerse fuera de las fronteras donde fueron producidas. Podríamos decir que la alimentación sirve como medio de movilización del imaginario que tenemos de nuestra comunidad. Estas identidades recuperan a la alimentación como una herramienta para cohesionar grupos, para demarcarlos de otros, que quedan excluidos del goce o posibilidad de alimentarse con algunos alimentos. Por ejemplo, el sabor de la tortilla, y la tortilla como un símbolo, es uno de los elementos simbólicos de lo mexicano. Aún ahora, ser mexicano es identificarse con el alimento, con la tortilla, a pesar de que la tortilla y sus derivados, ya son alimentos globales. Como argumenta Jeffrey Pilcher, la identidad, asociada con la autenticidad, como en el caso de los tacos, es una cuestión social creada, es maleable y ha sido contestada reiteradamente (Pilcher, 2012). La comida se convierte así no solo en un marcador de la nación, o de la región. Pertenecer a una región implica consumir y mantener una tradición gastronómica que se identifica como propia.

\section{Historias DE LA INDUSTRIALIZACIÓN}

Otro tema de la globalización es el impacto de la industrialización en los sistemas alimentarios. No podemos comprender el mundo culinario actual sin la alta tecnificación que hemos tenido en los 
últimos dos siglos. Pareciera que en este terreno, siempre pensamos en la industrialización como un elemento de hegemonía cultural, del norte imponiendo modelos hacia el sur, y pareciera ser que el intercambio entre los países latinoamericanos ha sido menor. En efecto, estos procesos no han sido tan ampliamente analizados por los historiadores, aunque esta situación ha empezado a cambiar.

Por ejemplo, podemos dar cuenta de unos intercambios interesantes entre los países del sur. La narrativa tradicional de la Revolución Verde de los años 1950’s y 1960's, toma la idea de cómo la Fundación Rockefeller y la Fundación Ford usaron la tecnología a su disposición, como algunas técnicas de hibridación de semillas, para aumentar la productividad agrícola, bajo el argumento antes mencionado de que se requería mayor producción de alimentos si se quería atacar el problemadela nutrición. Además, el uso defertilizantes y pesticidas acompañó al paquete tecnológico, para cederlas a los países del sureste asiático como parte de ayuda humanitaria para evitar las hambrunas. Fue tal el impacto, y la propaganda asociada al éxito de este proyecto, que el premio Nobel de la paz fue otorgado a Norman Borlaug en 1970, jefe del Programa en sus inicios. Sin embargo, algunas voces como Vandana Shiva o Nick Cullather, han cuestionado esta narrativa de "éxito" tecnológico, mostrando que si se consideran los impactos ambientales y sociales a mediano y largo plazo de esta Revolución, se debería cambiar la narrativa y cuestionar firmemente el supuesto beneficio de este programa biotecnológico de las fundaciones filantrópicas (Cullather, 2010; Shiva, 2000; Shiva et al., 2001).

También en la narrativa tradicional, se ofrece la idea del "norte" llegando al rescate del "sur", que debe ser matizado y cambiado profundamente. Sin embargo, si atendemos a los intercambios latinoamericanos, la historia que se puede contar es muy diferente. La semilla, valga la redundancia, de la Revolución Verde se encuentra en México, o por lo menos así deberíamos de entenderlo. A partir de 1943, se creó el Programa Agrícola Mexicano (PAM), dependiente de la División de Ciencias Naturales de la Rockefeller, en una zona cercana a la Ciudad de México, Chapingo. El Instituto 
Nacional de Nutriología, creado el mismo año y financiado por la Rockefeller y el gobierno mexicano, se encargó de hacer los análisis composicionales de los granos producidos. El PAM recuperó los conocimientos de los campesinos mexicanos, estudió las variedades locales, sembró y mejoró las semillas, en colaboración directa con ingenieros agrónomos locales. Esta historia entre México y Estados Unidos está siendo estudiada por varias personas, incluyéndome, sin embargo, hay un aspecto que ha sido poco estudiado, y que es el intercambio de las semillas con otros países de Latinoamérica: las semillas del MAP, antes de ser enviadas a Asia, fueron llevadas a Colombia, y a otros países de Latinoamérica. Sin embargo, este aspecto aún no es analizado por la historiografía y representaría un interesante punto de partida para historias interconectadas entre los países latinos, de cómo la industrialización ha afectado nuestra forma de alimentarnos y su impacto en la agricultura y nuestros modelos de consumo ${ }^{1}$.

Otra posibilidad, aún más remota temporalmente, es el caso del extracto de carne de Justus von Liebig. Liebig, quien es considerado uno de los fundadores de la química orgánica gracias a que perfeccionó metodologías para el análisis de alimentos, y realizó experimentos en los cuales estableció el actual modelo composicional, carbohidratos, lípidos y proteínas. La comercialización de sus productos (Liebig abogaba por una dieta alta en proteínas de origen animal) lo impulsó a tener relaciones comerciales con Argentina para la producción y comercialización de su extracto de carne (Finlay, 1995). Habría que hacer la historia de este intercambio, y explorar si hubo más de los cuales aún no estamos enterados.

Finalmente, entre estas historias de la industrialización de la producción agrícola y de alimentos, permea la idea d que juzga moralmente a la producción industrial. No podemos entender el mundo actual sin la abundancia de alimentos procesados, unos más que otros, para los cuales aún hacen falta historias de que nos den cuenta de los impactos de estos procesos industrializadores — de alguna manera, el trabajo de Mintz es precursor también de estas historias. Sin embargo, existe la idea de que la industrialización es 
inherentemente "mala" en sí, que altera el orden "natural" de los alimentos. Como se argumenta Rachel Laudan, la comida no es siempre natural, también es sometida a procesos, y la industrialización hay que comprenderla en su amplia complejidad (Laudan, 2016). Nuevos acercamientos de guías nutrimentales, como la Clasificación Nova (Monteiro, 2009) recuperan diferencias en los niveles de procesamiento de los alimentos, haciendo necesarios más trabajos de historia de la industrialización de alimentos para comprender el surgimiento de estas nuevas propuestas y cómo entenderlas en la larga historia de la industrialización

\section{HistoRIAS DE LA NUTRICIÓN Y LA MEDICALIZACIÓN}

Los procesos de industrialización de los últimos dos siglos son paralelos del surgimiento de la nutrición y de la medicalización de la alimentación. Uno de los aspectos de nuestra actual comprensión sobre los alimentos y la alimentación surge de lo que las prácticas, teorías y tecnologías médicas y científicas nos han brindado, y que hemos interpretado como realidades objetivas sobre la naturaleza de los alimentos y sus transformaciones en el fenómeno de la alimentación. A pesar de su importancia en la actualidad, aún hay pocos trabajos que exploren la historia de cómo surgió esta mirada medicalizada y tecnocientífica sobre la alimentación en los contextos latinoamericanos2, con excepciones y esfuerzos importantes, como los que veremos en las siguiente sección. Considero que esta perspectiva histórica debería incorporarse a los estudios sociales y culturales sobre la alimentación, entre otras cosas, porque brinda un contexto temporal sobre los procesos de medicalización contemporáneos. Estos procesos han sido invisibilizados en muchos de los estudios sobre alimentación, que enfatizan la parte cultural, social o antropológica, y que toman al conocimiento científico de la nutrición como un reflejo de la realidad, ocultando su carácter histórico, contingente, y situado, de ahí la importancia de destacar estas historias, para ver cómo llegamos a entender a la alimentación en los términos actuales.

En varios estudios se sigue reificando la idea de que las 
recomendaciones nutricionales, expuestas por los expertos, son "reflexiones objetivas de verdades nutricionales" cuando en realidad, de acuerdo con Charlotte Biltekoff, son "reflejo de ideales sociales", además de que incorporan varios elementos morales que han permanecido en la alimentación desde la antigüedad (Biltekoff, 2012,173). Este trabajo se inserta en lo que Biltekoff llama estudios críticos de la nutrición, dado que pretendo estudiar la nutrición desde la perspectiva de los estudios sociales e históricos sobre la ciencia, que muestra que en la nutrición como disciplina se han incorporado los compromisos sociales de quienes mantienen y crean los conceptos, estándares y guías nutricionales, así como los presupuestos de la sociedad en que son creados. Entre lo que se analiza en esta perspectiva es la imbricación de temas transdisciplinarios, como la enfermedad, la raza y la clase, temas que quizás son más notorios en contextos considerados no hegemónicos en lo referente a la producción de conocimiento científico (Pohl-Valero 2014, Vargas-Domínguez 2015). Enseguida analizaré, de manera muy breve, algunas historias locales sobre nutrición en América Latina, ahora desde los contextos nacionales, para ejemplificar la propuesta de esta historia de la nutrición crítica.

\section{HisTORIAS DE LA NUTRICIÓN EN AMÉRICA LATINA}

La historia de cómo se ha abordado la historia de la nutrición en los contextos latinoamericanos es interesante. Existen unas narrativas tradicionales sobre la nutrición, que fueron creadas por los actores que estuvieron involucrados en los hechos, o que representan historias institucionales que sirven como referencia obligada (Bourges R., 2002). La profesionalización de la historia de la ciencia ha incorporado nuevas preguntas y temas a la historia de la nutrición en América Latina. A continuación, haré un breve repaso a historias de la nutrición en algunos países, enfocándome en la primera mitad del siglo XX y en los actores que crearon algunas investigaciones sobre el tema y, en su caso, impulsaron la creación de instituciones locales especializadas. Hay que hacer una distinción antes de proceder. Existieron dos acercamientos a la nutrición. Uno, el clínico, se 
encargaba principalmente de los problemas asociados con la mala alimentación desde una perspectiva individual, tratando de dilucidar la etiología de las enfermedades, así como en su terapéutica. Otro acercamiento fue el de la nutrición social, que intentaba abordar los problemas de la alimentación desde una perspectiva poblacional, epidemiológica, en un acercamiento cercano a la salud pública y la medicina social. Ambos acercamientos estuvieron presentes en la primera mitad del siglo XX, como veremos más adelante.

\section{Argentina}

Empezaré por la Argentina, aunque no necesariamente este orden obedezca a una jerarquía. En Argentina se encuentra a uno de los actores principales de la nutrición en la región en los otros países, el médico Pedro Escudero (1887-1963). Recupero principalmente el trabajo de José Buschini, y su excelente investigación sobre el tema.

Pedro Escudero, desde 1924 propuso la creación de un instituto especializado en nutrición, que se concretó en 1928, con la creación del Instituto Municipal de las Enfermedades de la Nutrición, que como su nombre lo indicaba, se interesaba en el aspecto clínico (SCHOR, 2002). El interés de Escudero fue paulatinamente ampliando su alcance, de lo clínico, se desplazó hacia el estudio de los problemas socioeconómicos de la alimentación, con llamados a que se estudiara el aspecto económico y sanitario de la alimentación problema en el que se enfocó en su Instituto.

Para Escudero, la alimentación o más bien, la mala alimentación, tenía un efecto degenerativo en la sociedad argentina, y se valía de estadísticas para sostener sus dichos: de acuerdo con sus datos, un tercio de la población no comía lo suficiente para vivir en salud (Buschini, 2016,137). En este sentido, la alimentación "defectuosa" era parte de la degeneración por él percibida, y esto era un problema social antes que biológico. La opinión de Escudero, publicada en periódicos y divulgada ampliamente, facilitó que en 1934 se le aumentaran recursos a su Instituto y se lograra su autonomía y en 1938, se le dio carácter de nacional.

De acuerdo con Bsuchini, el contexto en que Escudero trabajó 
debemos asociarlo con dos miradas diferentes: por un lado, el estudio de la alimentación y consumo de los trabajadores en la Organización Internacional del Trabajo (OIT), y por otro lado, el enfoque biológico, de la Comisión de Salud de la Sociedad de Naciones3. Ambos enfoques Escudero los reunió, creando por un lado proyectos de Asistencia del Enfermo, con énfasis en la parte biológica o clínica, y por otro, el proyecto de Acción Social, que atendía la parte socioeconómica. En esta unión, el énfasis en la infancia fue crucial, dado que fue en este grupo que se vincularon fuertemente ambas perspectivas, y se crearon proyectos de apoyo a la madre y a los niños, con hospitales y comedores escolares, herencia de tradiciones como la puericultura. El Instituto realizó encuestas de ingresos, del tipo de alimentación y estado de salud, para conocer el estado "orgánico" y "económico-social" de las familias, en Buenos Aires, Mendoza y Avellaneda. El resultado de estos y otros estudios, le permitieron a Escudero relacionar el precio de los alimentos, los salarios y el costo de una canasta alimentaria que siguiera las cuatro leyes de la alimentación que había propuesto Escudero -Alimentos en buena Cantidad, Calidad, en Armonía-Equilibrio para el cuerpo, y Adecuados al individuo y la sociedad. Gracias a los trabajos de Escudero, se podía saber si el salario alcanzaba para una dieta adecuada, o no lo hacía. Los estudios mostraron que buena parte de los trabajadores no ganaban lo suficiente, y al parecer, sus ideas orientaron a la acción social (Buschini, 2016).

De acuerdo por lo recuperado por Buschini, para 1945, al finalizar al Guerra Mundial, el Instituto de Escudero reunía 385 miembros. Comparándolo con cualquier otro instituto de nutrición en Latinoamérica, en la época era el más grande centro de especialidades de nutrición. En sus proyectos se mantuvo el espíritu formador, vinculado a la necesidad de tener una base de profesionales de la nutrición que pudieran realizar el cambio social por él deseado. Escudero formó Dietistas desde 1935, y en 1938 se creó una Clínica de la Nutrición en la Facultad de Ciencias Médicas de Buenos Aires. Fue en esta Clínica que se impulsó la creación de un programa de becas para médicos de otros países. Este programa 
fue aprovechado por varios médicos de Bolivia, Brasil, Colombia, Chile, México, Paraguay y Uruguay (Buschini, 2016). También, desde su posición, se vinculó con la Sociedad de Naciones para que se realizara en Buenos Aires la Tercera Conferencia Internacional de Nutrición en 1939, con asistentes de diversos países y observadores de la OIT (Pernet, 2013,256). Con la llegada de Perón en 1940, el INN fue centralizado y perdió autonomía y fuerza, y Escudero renunció a su cátedra y la dirección del INN en 1946, alejándose de la nutrición y su aspecto social(López, 2012).

Como un caso particular, pero que entra en la discusión de la nutrición en Argentina, en 1945, el Ministerio de Salud de Bolivia invitó a Pedro Escudero a llevar a cabo encuestas de nutrición en las minas del país, lo cual, al parecer, tuvo un impacto en el diseño de las políticas públicas de alimentación, con la sugerencia de la creación de instituciones especializadas en Nutrición, sin embargo, estas no fueron creadas sino hasta 1958, con la Dirección Nacional de Nutrición y Alimentación. El rol de Escudero en las políticas públicas de Bolivia aún no ha sido completamente investigado (San Miguel S., 2002; Schor, 2002).

BRASIL

En Brasil, el estudio de la nutrición fue impulsado por los médicos desde finales del siglo XIX, pero se consolidó en el movimiento sanitarista de la Primera República de inicios del 1930, principalmente en Sao Paulo y Rio de Janeiro. Como en el resto de los países latinoamericanos, dos corrientes persistían: la perspectiva clínica (con énfasis en la fisiología y la endocrinología) y la perspectiva social, influenciada por los estudios del argentino Pedro Escudero.

Es difícil hacer un recuento histórico de un país tan grande y diverso como Brasil que tuvo muchas formas de enfrentarse a lo que se conoció como el problema de la nutrición, para lo cual me baso en los trabajos de José Arimatea Barros Bezerra y de Francisco de Assis Guedes de Vasconcelos (Bezerra, 2012; Vasconcelos, 2001). El estudio de la nutrición en Brasil, hacia las décadas de 1930 y 
1940, fue compartido no solo por los médicos, sino también por un creciente número de antropólogos y sociólogos, como Gilberto Freyre, Jamesson Ferreira Lima, Josué de Castro, Nelson Chaves, entre otros, quienes se alejaron de los dos grandes centros urbanos, Sao Paulo y Río de Janeiro, y llevaron a cabo importantes investigaciones en el Pernambuco. Lo mismo que en la mayoría de los países latinoamericanos, dos intereses guiaron la investigación: la erradicación del hambre de la población local, considerada en pobreza, y la pretensión de modificar la alimentación de la población con fines eugenésicos (Vasconcelos, 2001).

El trabajo de Gilberto Freyre, de acuerdo con Vasconcelos, reiteraba la idea de que se podía mejorar la raza brasileńa, el mestizo, a través de cambios en la alimentación. De acuerdo con Freyre, la harina de mandioca, uno de los principales alimentos del pueblo brasileńo, era de menor calidad que la harina de trigo, por tener un menor contenido de proteínas, reiterando la idea de que los alimentos americanos eran de menor valía y calidad que los alimentos del Viejo Mundo (Vasconcelos, 2001,319-320). Para salir de la opresión social, Freyre proponía que los campesinos del Pernambuco, dedicados en buena medida al cultivo de cańa de azúcar, Freyre proponía el abandono del monocultivo latifundista.

La perspectiva de Freyre, cercana a la nutrición social, resultaba errada para Josué de Castro, quien desde una perspectiva más biológica, más clínica que Freyre, consideraba que era necesario el estudio de las raciones de los trabajadores, para conocer el consumo y posteriormente, delimitar el salario mínimo, pero basándose en estudios científicos más claros que los empleados por Freyre. Castro creó en 1940 el Servicio de Alimentación de Asistencia Social, el Servicio Técnico de Alimentación (1941) y la Comisión Nacional de Alimentación en 1945 y el Instituto de Nutrición de la Universidad de Brasil en 1946. La idea del motor humano, idea que provenía desde finales del siglo XIX, aplicada a los trabajadores brasileños era clara: para Castro. Para él a pesar de que los trabajadores consumían suficiente combustible, medido en calorías, requerían mayor cantidad de proteínas en su dieta. Castro, como Nelson 
Chaves, proponían la vinculación entre pobreza y enfermedad, con la diferencia de que Castro se acercaba desde una perspectiva más social que Chaves, más biológico o clínico.

Podemos ver en estos tres representantes de la élite intelectual brasileña dedicada al estudio de la alimentación, una gradación entre las aproximaciones sociales, de Freyre, hacia las más clínicas, como las de Chaves, cercanas a lo que se consolidó en la segunda mitad del siglo XX. Castro representa el típico científico nutricionista del periodo, con una mezcla de ambas perspectivas. Los diversos acercamientos a la comprensión y solución del problema de la nutrición quizás se deba los orígenes sociales de estos científicos, Castro descendía de agricultores, y Freyre y Chaves, provenían de de familias aristocráticas. Castro defendió que lo que aquejaba al pueblo brasileño no era una cuestión de raza, sino de hambre. El hambre, reiteró Castro en su libro de Geografía del hambre, debía mantenerse como guía, y no dejarlo todo en el estudio de la malnutrición, que ocultaba el origen social del problema. Para Castro, había que mejorar las condiciones socioeconómicas de la población. Similares acercamientos existieron en otros países.

\section{Chile}

El surgimiento de instituciones centradas en la nutrición en Chile mantiene tendencias en común con los otros países de la región. Un médico, Eduardo Cruz-Coke, es reconocido por su trabajo pionero sobre las vitaminas y es considerado uno de los principales impulsores de la nutrición en Chile. Sus discípulos se acercaron al problema de la nutrición desde una perspectiva poblacional, destacando entre ellos Jorge Mardones-Restat, quien creó junto con Cruz Coke el primer Consejo Nacional para la Alimentación y Nutrición de Chile en 1937, quienes se interesaron no solo en conocer el estado nutricional de la población, sino que tuvieron un especial interés en el conocimiento de la alimentación de los obreros.

Como ha mostrado Juan Carlos Yánez, la alimentación desde una perspectiva científica se venía estudiando desde el siglo XIX, impulsada con la creación de las Gotas de Leche para mujeres 
embarazadas, niños y bebés, bajo la dirección de Luis Calvo Mackenna (Yáńez Andrade, 2017). Es decir, desde la puericultura o el mejoramiento de la sociedad a través de la alimentación infantil, se intentó modificar a la población chilena. La alimentación se consideraba una herramienta que podía ser empleada para mejorar a la raza, a través de la comprensión de los cuerpos como motores que, alimentados adecuadamente, podían cambiar, ser mejorados. La fuerte tendencia eugenésica de varios personajes en la década de 1930 e inclusive hasta bien entrado el siglo XX, guiaron la investigación médica sobre la alimentación pensando en el influjo del medio ambiente sobre los cuerpos, o justificando estas nociones haciendo uso de las metáforas termodinámicas de la época (Sánchez Delgado, 2018).

Sin embargo, para implementar estas estrategias era necesario conocer con mayor claridad el consumo de alimentos de la población, así como el costo de los alimentos, dado que se entendía que los nutriólogos debían interesarse en el aspecto socioeconómico. Con este fin, en 1928, la Dirección General de Estadística realizó encuestas sobre la alimentación de la población con el fin de determinar el Índice del Costo de Vida de los habitantes de Santiago. Posteriormente, en 1931, se llevó a cabo el Primer Congreso de Alimentación Popular en Valparaíso, en donde, tras una serie de debates, en las conclusiones de la reunión se proponía la creación de instituciones dedicadas al estudio de la alimentación, con el fin de proponer mecanismos para modificar la alimentación de la población, siendo uno de ellos la educación en el terreno de la nutrición. Además, los congresistas reiteraron la necesidad de conocer el consumo de los alimentos y el costo de estos, para con ello delimitar su propuesta de un "sobre salario familiar", y con ello atender a las causas económicas de la mala alimentación. Diversos actores se interesaron en estos temas, entre ellos encuestadores de la Sociedad de Naciones y de la OIT quienes también diagnosticaron que el principal problema en Chile era el de la alimentación, problema que se asociaba con los bajos salarios (Yáñez Andrade, 2018). Es decir, la nutrición se consideró no solo un problema fisiológico o bioquímico o meramente clínico, sino 
también un problema socioeconómico, es decir, estas tendencias las podríamos insertar fácilmente en el terreno de la nutrición social.

Con la llegada de la Gran Depresión en 1929, el interés se amplió a mejorar las condiciones de vida de toda la población, no solo de los trabajadores o de los niños. La pobreza, aunque aún no se delimitaba ni se cuantificaba como lo hacemos ahora, se empezó a vincular con la incapacidad de la gente de tener una alimentación adecuada. Las encuestas se ampliaron a otros grupos y otras ciudades, por ejemplo, en 1933 se observó que el $80 \%$ de las familias de trabajadores de una refinería de azúcar en Valparaíso no consumían lo suficiente de acuerdo con el trabajo realizado, a pesar de estar en mejores condiciones laborales y salariales que otros obreros (Yáñez Andrade, 2017).

La creación del Consejo de Alimentación de 1937 fue en sí la institucionalización de los diversos proyectos de alimentación existentes en Chile, pero con la finalidad de delimitar un programa político, originado desde los médicos, que incidiera en las políticas locales para aumentar los salarios. Los investigadores en nutrición local se interesaron en la solución de problemas socioeconómicos, porque al abordarlos, se argumentaba, mejorarían los problemas asociados a la mala alimentación, además de que se frenaría el deterioro de la raza. Como en otros países de Latinoamérica, la alimentación en Chile también fue considerada una herramienta eugenésica en la época.

\section{Colombia}

Como ha mostrado Stefan Pohl Valero para el caso colombiano, diversos mecanismos se implementaron en Colombia para optimizar la productividad de los trabajadores, para regenerar el cuerpo trabajador, enmarcados en una racialización de la sociedad colombiana. Entre más blanco y europeo, mejor. Y se argumentó que la alimentación era uno de los aspectos del medio ambiente que influían en este mejoramiento racial. Este mejoramiento tuvo la peculiaridad de estar fuertemente asociado al clima, cuya diversidad, propiciada por la orografía local, tenía una influencia, en la mayoría 
de las veces negativa, sobre los cuerpos, y una de las formas de contrarrestar este influjo era a través de una alimentación racional que siguiera los dictados de la ciencia moderna (Pohl-Valero, 2015).

A inicios del siglo XX era de uso común el argumento de la necesidad de esta alimentación racional, lo cual se lograría gracias a los proyectos educativos, en los cuales se privilegió el consumo de alimentos adecuados, como la carne, y se propuso que se abandonara el consumo de otros que se asociaron con la degeneración de las clases populares, como la chicha, una bebida fermentada de maíz, que se pensaba producía una enfermedad que debilitaba a los trabajadores, el chichismo (Pohl-Valero, 2016). Abandonar rasgos atávicos podía lograrse por medio de la educación, además de que también serviría como medio para que las clases populares pudiesen comprar los mejores alimentos de acuerdo con el salario percibido. Además, el consumo de ciertos alimentos como la carne se vinculó con características deseadas, como la inteligencia y la valentía, y su ausencia con los caracteres indeseados, como debilidad y pereza. Estas asociaciones recuperaban la noción del cuerpo humano como un motor, que, al regular y mejorar su alimentación, podía aumentar su productividad. Estas nociones produjeron lo que PohlValero llama "termodinámica social", que se convirtió en una de las herramientas eugenésicas para la mejoría de la población (PohlValero, 2016; Pohl-Valero, 2014).

Estas herramientas eugenésicas se insertaron en discursos asociados con la modernización y eficiencia del Estado, que ocurriría a través de los trabajadores, quienes, desde finales del siglo XIX, se convirtieron en objeto de estudio. Ya en la primera mitad del siglo XX, el Ministerio de Higiene, Trabajo y Previsión Social, entre 1930 y 1940, bajo la dirección del Dr. Jorge Bejarano, publicó cartillas de la alimentación de la clase obrera, y guías para la alimentación de los trabajadores y los niños. Se debían mejorar las condiciones socioeconómicas de la clase obrera para que tuvieran un mejor consumo. Entre mejor fuese la alimentación, mejor sería la eficiencia del motor humano. Se entendía por "mejor" un consumo elevado de leche y carne. Las Gotas de Leche, entre otros proyectos iniciados 
en Colombia desde los años 1930's, enfatizaron la alimentación de los niños, y posteriormente le siguieron los comedores escolares, pensando en el tema de la puericultura como eje central de mejoramiento racial y biológico, cambio que se acompañaría de cambios sociales. Para Bejarano, lo mismo que para el resto de encargados de las investigaciones sobre alimentación, lo social iba de la mano de lo meramente biológico (Pohl-Valero, 2016).

Los expertos colombianos también se insertaron en las dinámicas de los organismos internacionales, como la Sociedad de Naciones, la Organización Internacional del Trabajo, y la Oficina Sanitaria Panamericana. Los datos presentados en dichos espacios eran fruto de las encuestas locales, como por ejemplo las elaboradas por José Francisco Socarrás, quien empleó "la cuantificación para dar cuenta de lo social", reiterando la vinculación entre el cuerpo del trabajador, la productividad, los salarios y la alimentación (PohlValero, 2016,143).

A pesar de estos acercamientos con un profundo interés social al estudio de la nutrición en Colombia, las historias más tradicionales omiten esta perspectiva. La historia institucional inicia en 1944 con la creación del Laboratorio de Bromatología, antecedente de lo que sería el Instituto Nacional de Nutrición creado en 1947. Estos acercamientos otorgan un mayor peso al estudio de la nutrición desde la perspectiva clínica o bioquímica, olvidando este rico pasado de una nutrición social poderosa en Colombia.

\section{MÉxico}

En México, el estudio de la nutrición tuvo una fuerte impronta eugenésica, que provenía de diversas fuentes. Por un lado, la alimentación de la mayor parte de la población era entendida como de inferior calidad al mezclarse prejuicios racializados en contra de lo indígena. De acuerdo con la narrativa nacionalista, la "raza cósmica" de Vasconcelos, es decir, los mestizos, alcanzarían todo su potencial al adoptar patrones de consumo basados en la alimentación modelo europea o estadounidense. La influencia francesa en la medicina de finales del XIX y el creciente influjo de la estadounidense a inicios 
del XX, crearon una medicina que se interesaba en el salubrismo, en la puericultura, y también en la fisiología, y la alimentación formaba parte de este conglomerado que formaba parte de los proyectos de salud pública. Los médicos mexicanos tuvieron acercamientos con la eugenesia anglosajona, de manos de las fundaciones filantrópicas como la Fundación Rockefeller y la Carnegie Institution y sus proyectos insertos en México (Vargas-Domínguez, 2015; VargasDomínguez, 2016). También, existió la vinculación con la eugenesia latina, representada por Corrado Ginni, el eugenecista italiano, quien estudió las poblaciones "primitivas" y "puras" indígenas, realizando estudios en México, con influencia en las corrientes antropológicas mexicanas de la década de 1930 (Berlivet, 2016). Todas estas aproximaciones, compartían la idea de que se podían atacar los problemas sociales a través del cambio en las características de la población, para lo cual, la alimentación era uno de los elementos a mejorar, generando aproximaciones por fisiólogos, biólogos, y médicos.

Las múltiples investigaciones sobre el tema de la alimentación intentaron ser cohesionadas en un solo organismo, y en 1936, se creó la Comisión Nacional de Alimentación, encargada de estudiar las condiciones de la alimentación de los grupos más marginados, y que habían sido visibilizados por el conflicto revolucionario, los indígenas y los campesinos. Estos dos grupos, se sumaron al otro problema del Estado, este más viejo aún, los pobres urbanos. Pobres, campesinos e indígenas, compartían el problema de que su alimentación se consideró deficiente. La idea de que la alimentación tradicional era deficiente se mantuvo durante toda la primera mitad del siglo XX, y diversos estudios, entre ellos encuestas en zonas indígenas marginadas, o en las zonas más pobres de la ciudad de México, confirmaron la hipótesis que el consumo de proteínas animales era muy inferior a lo demarcado por los estándares internacionales (Vargas Domínguez, Aceptado para publicación el 9 de octubre de 2018).

En 1943, se creó el Instituto Nacional de Nutriología, que pensó en atacar el problema de la nutrición por medio de entender el 
vínculo entre pobreza y mala alimentación. Su director, Francisco de Paula Miranda, fue un impulsor de tratar de mejorar a la población pobre a través de una alimentación de bajo costo, convencido de que lo que hacía falta era un salario adecuado. Uno de los investigadores del Instituto, José Quintín Olascoaga había publicado entre 1939 y 1940, un artículo extenso sobre la nutrición. Sus indicaciones eran retomadas de la Sociedad de Naciones, donde exponía que un trabajador debía consumir alrededor de 3500 calorías. Si se quería resolver el problema de la nutrición, debía atacarse el problema social de los bajos salarios para garantizar que le fuera repuesta la energía gastada en la jornada. La perspectiva de Olascoaga estaba orientada por su estancia por cerca de dos años en el Instituto de Nutrición de Escudero. Esta orientación social, explicó la mala alimentación como un problema socioeconómico, no racial ni biológico. La alimentación de los mexicanos era mala porque no tenían para comer. Los estudios de Olascoaga fueron retomados por diversos actores para exigir mejoras a los salarios, para evitar el hambre. También en Nutriología se realizaron los estudios bioquímicos de las semillas que se produjeron en el Programa Agrícola Mexicano, origen de la Revolución Verde, como expliqué en la primera sección. Estas colaboraciones fueron fruto de las redes de investigación que se tejieron al interior de Nutriología, siendo Miranda el representante mexicano ante la Organización para la Agricultura y la Alimentación de las Naciones Unidas (FAO) en sus primeros años (Vargas-Domínguez, 2014).

La perspectiva del Instituto Nacional de Nutriología obedece a lo que llamamos nutrición social. En contraste, otras instituciones, como el Hospital de Enfermedades de la Nutrición a cargo de Salvador Zubirán, o el Hospital Infantil de México, a cargo de Federico Gómez o el Instituto Nacional de Cardiología, a cargo de Ignacio Chávez, se acercaron al estudio de la nutrición desde una perspectiva clínica, enfatizando el estudio de las enfermedades asociadas a la mala alimentación. Ambas perspectivas se combinaban, no eran excluyentes, y se realizaron trabajos colaborativos entre las distintas instituciones durante las décadas de 1940 y 1950, y fue a 
partir del cierre de Nutriología que la perspectiva social pasa a un segundo plano en el interés de los investigadores.

\section{Consideraciones finales}

Después de esta breve exposición de dónde se inserta la historia de la nutrición en los estudios sobre alimentación, además de este acercamiento panorámico a algunas historias nacionales de la nutrición, que no intenta ser exhaustiva, surge la pregunta de ¿cómo podemos entender el estudio de la nutrición en Latinoamérica? ¿Se puede entender únicamente como una disciplina en la cual la influencia estadounidense y europea marcaron la pauta y los actores locales siguieron sus dictados? ¿O podemos entenderla como un proceso más complejo, más rico, si vemos las similitudes de los casos nacionales?

Lisa Roberts ha criticado la mirada reduccionista que se obtiene si nos enfocamos únicamente en la dicotomía "el Occidente y los demás" [the West and the rest](Roberts, 2010,20). Los estudios poscoloniales han criticado fuertemente esta mirada, en la cual dos culturas —en el caso de la nutrición podríamos hablar de culturas nutricionales locales - pueden intercambiar el conocimiento, pero siempre manteniendo la premisa de que son dos culturas o contextos con diferencias sustanciales entre ellos, de una manera muy parecida a lo que se hace cuando hablamos de intercambios Norte-Sur (Raj 2013, 2007), hablamos de una hegemonía entre un sitio u otro(Raj, 2013). Las historias de encuentros que mencioné en la primera sección, pueden ser sujeto de estas críticas, sin embargo, trabajos como el de Marcy Norton responden a la crítica y orientan hacia nuevas líneas de investigación que complejizan la narrativa histórica.

Quienes hemos hecho historia de la nutrición desde una perspectiva que podríamos llamar transnacional, nos hemos enfocado en mayor o menor medida en las relaciones ya sean entre los países considerados hegemónicos, con la circulación de estándares e instrumentos (Neswald et al., 2017), o analizando el fenómenos desde esta crítica poscolonial, enfatizando el carácter colonial de la nutrición, en donde recuperamos el poder explicativo de estas redes 
en los desarrollos locales. (Cwiertka et al., 2015; Pernet, 2014; Pernet, 2013; Pernet and Ribi Forclaz, 2018; Vargas-Domínguez, 2014). En estos trabajos, enfatizamos relaciones, entre Europa y los Estados Unidos y los países latinoamericanos. Actores como la Sociedad de Naciones, la Fundación Rockefeller, la FAO y la OMS, así como la Organización Internacional del Trabajo aparecen como actores que dictan o explican las tendencias. Recientes aportaciones a estas ideas transnacionales(Carter, 2018), realzan los fuertes y nutridos intercambios entre los países latinoamericanos y los organismos internacionales, siendo estos últimos los que parecen haber ejercido mayor influencia sobre los primeros. Sin embargo, aún queda por explorar cómo y en qué grado los desarrollos locales influenciaron a los organismos internacionales. Quizás lo que hemos visto quienes hacemos historia de la nutrición es fruto de las contingencias materiales: en la mayoría de los archivos locales —al menos en el caso de México así es - las fuentes relacionadas con el tema se encuentran fragmentadas, o han sido olvidadas en los múltiples cambios institucionales que se han realizado. Para hacer historia de la nutrición, construimos nuestras narrativas utilizando fuentes internacionales, principalmente de los organismos que tuvieron relación con nuestros países, como la Fundación Rockefeller o los organismos de las Naciones Unidas. Esta ausencia de la memoria histórica debe estar más presente en nuestras reconstrucciones y debemos hacer un análisis más crítico de nuestras fuentes.

A pesar de este problema, si analizamos tendencias en común, actores históricos que intercambian saberes, preocupaciones, instrumentos, formas de entender el problema de la nutrición, podríamos contar otras historias, salirnos de la necesidad de explicar todo desde una mirada desde el Norte y podríamos observar y analizar los intercambios entre nuestros países. Los estudios que mostré, de los cuales evidentemente hice una libre interpretación, ya tienen en si el germen de una vinculación entre los países latinoamericanos mayor de lo que se puede observar de manera aislada. Procesos comunes como el interés en mejorar a la población a través de la alimentación, que tuvo orígenes en la eugenesia y la puericultura 
es uno de ellos. Explorar en mayor profundidad cómo el pensar a los alimentos locales como degenerados siguió guiando nuestras prácticas científicas y conductas alimenticias es otro. Otra línea posible de investigación es el proceso de auge de la nutrición social en contraste con una perspectiva clínica o bioquímica, tema que no ha sido abordado aún por la historiografía en general. El interés en pensar que la pobreza era el origen de la mala alimentación es otra línea que guía la investigación en casi todos nuestros países. Las políticas públicas de alimentación obedecieron de alguna manera a la vinculación entre pobreza, salarios y la idea de una alimentación insuficiente o inadecuada., degenerada. Otra posible vía de investigación es el origen de los recursos económicos para el estudio de la nutrición en nuestros países, que brindaría importantes pistas sobre las redes de conocimiento locales. Asimismo, hace falta vincular y problematizar a la nutrición como ciencia en otras narrativas historiográficas, y no dar por hecho el conocimiento científico de forma acrítica. Los estudios críticos de la nutrición apelan esto.

Recientes trabajos, pioneros en recopilar historias de la nutrición locales (Yáñez Andrade, 2018), representan esfuerzos interesantes en la construcción de historias del tema más densa, que permitirían analizar fenómenos en conjunto. Nuevos proyectos han creado plataformas que consolidan a investigadores y temas (Rehsnal, 2018), permitiendo mejorar la vinculación y juntar en un solo sitio, la más reciente producción historiográfica sobre el tema, incrementando la posibilidad de estudios comparados Es por ello que podemos decir que ya existe la masa crítica para abordar la historia de la nutrición en perspectiva comparada. Hacer una historia de la nutrición desde Latinoamérica es una necesidad y una deuda que debemos saldar, si queremos salir de las historias globales que no retoman a Latinoamérica en su rica complejidad y diversidad. 
BibliografíA

"Bases para una nutrición correcta." Boletín de la Oficina Sanitaria Panamericana, vol. 19, no. 3, 1940, pp. 278-280.

AGUILAR Rodríguez, S. "Alimentando a la nación: género y nutrición en México (1940-1960)." Revista de Estudios Sociales, no. 29, 2008, pp. 28-41.

BARONA, J. L. "Nutrition and Health. The International Context During the Inter-war Crisis." Social History of Medicine, vol. 21, no. 1, 2008, pp. 87-105, doi:10.1093/shm/hkm114.

BERLIVET, L. A. "A laboratory for Latin eugenics: the Italian Committee for the Study of Population Problems and the international circulation of eugenic knowledge, 1920s-1940s." Historia, Ciencias, Saúde--Manguinhos, vol. 23, no. Suppl. 1, 2016, pp. 51-72.

BEZERRA, J. A. B. "Educação alimentar e a constituição de trabalhadores fortes, robustos e produtivos: análise da produção científica em nutrição no Brasil, 1934-1941.” História, Ciências, Saúde-Manguinhos, vol. 19, no. 1, 2012, pp. 157179.

BILTEKOFF, C. "Critical Nutrition Studies." The Oxford Handbook of Food History, edited by Jeffrey Pilcher, Oxford University Press, 2012, pp. 172-190.

Eating right in America : the cultural politics of food and health. Duke University Press, 2013.

BOURGES R., H. B. M., José; O’Donell, Alejandro M. . "Historias de la Nutrición en América Latina." Sociedad Latinoamericana de Nutrición; Fundación CAVENDES; Instituto Nacional de Ciencias Médicas y Nutrición Salvador Zubirán (INCMNSZ); Centro de Estudios sobre Nutrición Infantil, 2002.

BUSCHINI, J. "La alimentación como problema científico y objeto de políticas públicas en la Argentina: Pedro Escudero y el Instituto Nacional de la Nutrición, 1928-1946." Apuntes, no. 79, 2016, pp. 129-156.

CARTER, E. D. "Social medicine and international expert networks in Latin America, 1930-1945.” Global Public Health, 2018, 
pp. 1-12, doi:10.1080/17441692.2017.1418902.

CROSBY, A. W. The Columbian exchange; biological and cultural consequences of 1492. Greenwood Pub. Co., 1972. Contributions in American studies, no 2.

CULlATHER, N. "The Foreign Policy of the Calory." The American Historical Review, vol. 112, no. 2, 2007, pp. 337364, http://www.historycooperative.org/journals/ahr/112.2/ cullather.html.

The Hungry World: America's Cold War Battle against Poverty in Asia. Harvard University Press, 2010.

CWIERTKA, K. J. et al. "Editorial Introduction : Writing Global Food History." Global Food History, vol. 1, no. 1, 2015, pp. 5-12, doi:10.1080/20549547.2015.11435409.

DRINOT, P. La seducción de la clase obrera: trabajadores, raza y la formación del estado peruano. Instituto de Estudios Peruanos : Ministerio de Cultura, Viceministerio de Interculturalidad, 2016. Serie Peru problema,, vol. 44.

EARLE, R. The Body of the Conquistador: Food, Race and the Colonial Experience in Spanish America, 1492-1700. Cambridge University Press, 2012.

"'If you eat their food ... " : diets and bodies in early colonial Spanish America." The American Historical Review, vol. 115, no. 3, 2010, pp. 688-713, doi:10.1086/ahr.115.3.688.

FINLAY, M. R. "Early marketing of the Theory of Nutrition: The Science and Culture of Liebig's Extract of Meat." The science and culture of nutrition, 1840-1940, edited by Harmke Kamminga and Andrew Cunningham, Rodopi, 1995, pp. vii, 344 p.

KAMMINGA, H. and A. CUNNINGHAM. The science and culture of nutrition, 1840-1940. Rodopi, 1995. Clio medica, vol. 32.

LAUDAN, R. "In praise of artificial food." aeon https://aeon.co/ ideas/artificial-food-has-made-human-civilisation-possible. Accessed 5 de diciembre 2018.

LEVENSTEIN, H. Paradox of Plenty: A Social History of Eating in Modern America, Revised Edition. University of California Press, 2003. 
LIU, H. From Canton Restaurant to Panda Express : a history of Chinese food in the United States. Rutgers University Press, 2015. Asian American studies today.

LÓPEZ, L. B. P., Susana. "Historia de la Nutrición en la Argentina: nacimiento, esplendor y ocaso del Instituto Nacional de la Nutrición.” Diaeta, vol. 30, no. 140, 2012, pp. 39-46.

MCKEE, F. "The popularisation of milk as a beverage during the 1930s." Nutrition in Britain. Science, scientists and politics in the twentieth century, edited by David F. Smith, Routledge, 1997, pp. 123-141.

MINTZ, S. W. Sweetness and power: the place of sugar in modern history. Viking, 1985.

MONTANARI, M. Medieval tastes : food, cooking, and the table. Columbia University Press, 2015.

MONTEIRO, C. A. "Nutrition and health. The issue is not food, nor nutrients, so much as processing." Public Health Nutrition, vol. 12, no. 5, 2009, pp. 729-731, Cambridge Core, doi:10.1017/S1368980009005291.

MUDRY, J. Measured Meals: Nutrition in America. State University of New York Press, 2009.

NESWALD, E. et al. "Setting nutritional standards : theory, policies, practices." University of Rochester Press, 2017.

NORTON, M. "Tasting Empire: Chocolate and the European Internalization of Mesoamerican Aesthetics." The American Historical Review, vol. 111, no. 3, 2006, pp. 660-691, doi:10.1086/ahr.111.3.660.

PERNET, C. A. "Between Entanglements and Dependencies: Food, Nutrition, and National Development at the Central American Institute of Nutrition (INCAP)." International organizations and development, 1945-1990, edited by Marc Frey et al., Palgrave Macmillan, 2014, pp. 101-125. Transnational History Series.

"Developing Nutritional Standards and Food Policy: Latin American Reformers between the ILO, the League of Nations Health Organization, and the Pan-American Sanitary Bureau." 
Globalizing social rights : the international labour organization and beyond, edited by Sandrine Kott et al., Palgrave Macmillan, 2013, pp. 249-261. Contributor biographical information https://www.loc.gov/catdir/enhancements/ fy1609/2012040924-b.html

Publisher description https://www.loc.gov/catdir/enhancements/ fy1609/2012040924-d.html

Table of contents only https://www.loc.gov/catdir/enhancements/ fy1609/2012040924-t.html.

PERNET, C. A. and A. RIBI FORCLAZ. "Revisiting the Food and Agriculture Organization (FAO): International Histories of Agriculture, Nutrition, and Development." The International History Review, 2018, pp. 1-6, doi:10.1080/07075332.2018. 1460386.

PILCHER, J. "Introduction." The Oxford handbook of food history, edited by Jeffrey Pilcher, Oxford University Press, 2012, pp. xvii-xxiii. Oxford handbooks.

PILCHER, J. M. Planet taco : a global history of Mexican food. Oxford University Press, 2012.

The sausage rebellion: public health, private enterprise, and meat in Mexico City, 1890-1917. University of New Mexico Press, 2006.

POHL-VALERO, S. "¿Agresiones de la altura y degeneración fisiológica? La biografía del "clima" como objeto de investigación científica en Colombia durante el siglo XIX e inicios del XX.” Revista Ciencias de la Salud, no. 13, 2015, pp. 65-83.

"Alimentación, raza, productividad y desarrollo. Entre problemas sociales nacionales y políticas nutricionales internacionales, Colombia, 1890-1950." Aproximaciones a lo local y lo global: América Latina en la historia de la ciencia contemporánea, edited by Edna María Suárez Díaz and Gisela Mateos, Centro de Estudios Filosóficos, Políticos y Sociales Vicente Lombardo Toledano, 2016, pp. 115-154.

"'La raza entra por la boca": Energy, Diet and Eugenics in Colombia, 1890-1940." Hispanic American Historical Review, 
vol. 94, no. 3, 2014, pp. 455-486.

RAJ, K. "Beyond Postcolonialism ... and Postpositivism: Circulation and the Global History of Science." Isis, vol. 104, no. 2, 2013, pp. 337-347, doi:10.1086/670951.

REHSNAL. "Red de Estudios Históricos y Sociales de la Nutrición y Alimentación en América Latina " Red de Estudios Históricos y Sociales de la Nutrición y Alimentación en América Latina https://www.redehsnal.com/. Accessed diciembre 2018.

REMEDI, F. Dime qué comes y cómo lo comes y te diré quién eres: Una historia del consumo alimentario en la modernización argentina. Córdoba, 1870-1930. Centro de Estudios Historicos Segreti, 2006.

ROBERTS, L. "Situating Science in Global History: Local Exchanges and Networks of Circulation." Itinerario, vol. 33, no. 1, 2010, pp. 9-30, Cambridge Core, doi:10.1017/ S0165115300002680.

SAN MIGUEL S., J. L. "La Nutrición en Bolivia y las Figuras Patricias Bolivianas." Historias de la Nutrición en América Latina, edited by Héctor; Bengoa M. Bourges R., José; O’Donell, Alejandro M. , Sociedad Latinoamericana de Nutrición; Fundación CAVENDES; Instituto Nacional de Ciencias Médicas y Nutrición Salvador Zubirán (INCMNSZ); Centro de Estudios sobre Nutrición Infantil, 2002, pp. 87110.

SÁNCHEZ DELGADO, M. "Alimentación y eugenesia, aproximaciones desde Chile: 1900-1956.” Gobernaresalimentar. Discursos, legislación y politicas de alimentación popular. Chile, 1900-1950, edited by Juan Carlos Yáñez Andrade, América en Movimiento Editorial; Centro de Investigación en Innovación, Desarrollo Económico y Políticas Sociales, 2018, pp. 53-82.

SCHOR, I. S., Saúl. "Nutrición Siglo XX: Bosquejo Histórico de la República Argentina." Historias de la Nutrición en América Latina, edited by Héctor; Bengoa M. Bourges R., José; O’Donell, Alejandro M. , Sociedad Latinoamericana de Nutrición; Fundación CAVENDES; Instituto Nacional de 
Ciencias Médicas y Nutrición Salvador Zubirán (INCMNSZ);

Centro de Estudios sobre Nutrición Infantil, 2002, pp. 57-86. SEN, A. Poverty and famines: an essay on entitlement and deprivation. Clarendon Press; Oxford University Press, 1981.

SHAPIN, S. "'You are what you eat': Historical changes in ideas about food and identity." Historical Research, vol. 87, no. 237, 2014, pp. 377-392, doi:10.1111/1468-2281.12059.

SHIVA, V. Stolen harvest : the hijacking of the global food supply. South End Press, 2000.

SHIVA, V. et al. An ecological history of food and farming in India. RFSTE/Navdanya, 2001.

SUREMAIN, C.-É. d. and E. KATZ. "Introducción: Modelos alimentarios y recomposiciones sociales en América Latina." Anthropology offood, no. 6, 2009, http://journals.openedition. org/aof/6432.

TURNER, K. L. How the other half ate : a history of working class meals at the turn of the century. University of California Press, 2014. California studies in food and culture.

VARGAS-DOMÍNGUEZ, J. "El metabolismo racial: estudios eugenésicos en Jamaica y Yucatán entre 1920 y 1940.” Revista Ciencias de la Salud, vol. 13, no. Historias alternativas de la fisiología en América Latina, 2015, pp. 85-103, doi:dx.doi. org/10.12804/revsalud13.especial.2015.06.

"Entre la nación y el mundo: la nutrición en México en la Conferencia de las Naciones Unidas sobre alimentación de 1943." Cuestión social, politicas sociales y construcción del Estado Social en América Latina en los siglos XIX y XX, edited by Mario Barbosa Cruz and Fernando Remedi, Universidad Autónoma Metropolitana-Cuajimalpa; Centro de Estudios Históricos Carlos Segreti, 2014, pp. 175-192.

"Nutrition and the Rockefeller Foundation in Mexico." Rockefeller Archive Center Research Reports, 2016, http:// rockarch.issuelab.org/resource/nutrition-and-the-rockefellerfoundation-in-mexico.html.

"El auge y declive del Instituto Nacional de Nutriología de 
México y su proyecto de nutrición social de 1943 a 1956." Historia Mexicana, Aceptado para publicación el 9 de octubre de 2018.

VASCONCELOS, F. d. A. G. d. "Fome, eugenia e constituição do campo da nutrição em Pernambuco: uma análise de Gilberto Freyre, Josué de Castro e Nelson Chaves." Historia, Ciencias, Saúde-Manguinhos, vol. 8, no. 2, 2001, pp. 315-339.

VEIT, H. Z. Modern food, moral food : self-control, science, and the rise of modern American eating in the early twentieth century. University of North Carolina Press, 2013.

YÁNEZZ ANDRADE, J. C. "El Primer Congreso de Alimentación Popular (Valparaíso, 1931). Debates y Propuestas.” Gobernar es alimentar. Discursos, legislación y politicas de alimentación popular. Chile, 1900-1950, edited by Juan Carlos Yáñez Andrade, América en Movimiento Editorial; Centro de Investigación en Innovación, Desarrollo Económico y Políticas Sociales, 2018, pp. 39-52.

"El problema de la alimentación: un enfoque desde las encuestas de nutrición (Chile, 1928-1938)." América Latina en la historia económica, vol. 24, 2017, pp. 66-97, http:// www.scielo.org.mx/scielo.php?script=sci_arttext\&pid=S140522532017000100066\&nrm=iso.

Gobernar es alimentar. Discursos, legislación y politicas de alimentación popular. Chile, 1900-1950. América en Movimiento Editorial; Centro de Investigación en Innovación, Desarrollo Económico y Políticas Sociales, 2018.

ZAZUETA, M. d. P. "Milk against Poverty: Nutrition and the Politics of Consumption in Twentieth Century Mexico." School of Arts and Sciences, vol. Doctor of Philosophy, Columbia University, 2011. general editor, Pablo Picatto.

Notas de PÁgina

${ }^{1}$ Los datos de la extensión del PAM en otros países aparecen en un reporte interno de la Fundación Rockefeller, Rockefeller Archive 
Center, Rockefeller Foundation Collection, Record Group 3.1, Series 908, Box 14, Folder 145.

${ }^{2}$ Existen ya varios trabajos que recuperan el estudio de la nutrición, principalmente en los Estados Unidos y Europa, pero aún falta una visión más amplia sobre la historia de la nutrición desde otros contextos. Resaltan los trabajos de BARONA, J. L. "Nutrition and Health. The International Context During the Inter-war Crisis." Social History of Medicine, vol. 21, no. 1, 2008, pp. 87-105, doi:10.1093/shm/hkm114, ibid., BILTEKOFF, C. Eating right in America : the cultural politics of food and health. Duke University Press, 2013, CULLATHER, N. "The Foreign Policy of the Calory." The American Historical Review, vol. 112, no. 2, 2007, pp. 337-364, http://www.historycooperative.org/journals/ahr/112.2/cullather. html, KAMMINGA, H. and A. CUNNINGHAM. The science and culture of nutrition, 1840-1940. Rodopi, 1995. Clio medica, vol. 32, MUDRY, J. Measured Meals: Nutrition in America. State University of New York Press, 2009, NESWALD, E. et al. "Setting nutritional standards : theory, policies, practices." University of Rochester Press, 2017, VEIT, H. Z. Modern food, moral food : self-control, science, and the rise of modern American eating in the early twentieth century. University of North Carolina Press, 2013.

${ }^{3}$ Podríamos matizar esta división, dado que al interior de la Sociedad de Naciones convivieron los dos acercamientos a la nutrición, el biológico y el social. Buschini llama biológico al acercamiento clínico. Sin embargo, desde este acercamiento biológico hubo dos aproximaciones: por un lado el acercamiento clínico, y por otro, el acercamiento bromatológico, es decir, de análisis de alimentos. El análisis de alimentos tenía una larga tradición, y era empleado tanto por quienes recuperaban la nutrición social como quienes se acercaban desde el aspecto clínico, por lo que habría que clarificar.

Fecha de Recepción del Artículo: 15 de marzo de 2018 Fecha de Aprobación: 22 de Julio de 2018 